\title{
Dual Modulation of Cyclooxygenase and CYP Epoxygenase Metabolism and Acute Vascular Inflammation in Mice
}

\author{
Akinyemi Oni-Orisan ${ }^{a,}{ }^{,}$, Yangmei Deng ${ }^{a}{ }^{*}$, Robert N. Schuck ${ }^{\mathrm{a}}$, Katherine N. Theken ${ }^{\mathrm{a}}$, \\ Matthew L. Edin ${ }^{b}$, Fred B. Lih ${ }^{b}$, Kimberly Molnar ${ }^{\mathrm{a}}$, Laura DeGraff ${ }^{\mathrm{b}}$, Kenneth B. Tomer ${ }^{\mathrm{b}}$, \\ Darryl C. Zeldin ${ }^{\mathrm{b}}$, and Craig R. Lee ${ }^{\mathrm{a}}$ \\ aDivision of Pharmacotherapy and Experimental Therapeutics, Eshelman School of Pharmacy, \\ University of North Carolina, Chapel Hill, NC, 27599, United States \\ bLaboratory of Respiratory Biology, Division of Intramural Research, National Institute of \\ Environmental Health Sciences, National Institutes of Health, Research Triangle Park, NC, \\ 27709, United States
}

\begin{abstract}
Cyclooxygenase (COX)-derived prostaglandins and cytochrome P450 (CYP) epoxygenasederived epoxyeicosatrienoic acids are important regulators of inflammation; however, functional interactions between these pathways in the regulation of vascular inflammation in vivo have not been studied. We investigated the relative and additive effects of endothelial CYP2J2 overexpression (Tie2-CYP2J2-Tr), global sEH disruption (Ephx $2^{--}$), and pharmacologic COX inhibition with indomethacin on the acute vascular inflammatory response to endotoxin in mice. Compared to vehicle-treated wild-type C57BL/6 controls, induction of myeloperoxidase (MPO) activity in lung and liver was similarly attenuated in Tie2-CYP2J2-Tr mice, Eph $2^{--1}$ mice and wild-type mice treated with moderate dose indomethacin. Dual modulation of both pathways, however, did not produce an additive anti-inflammatory effect. These findings demonstrate that both COX and CYP epoxygenase-mediated eicosanoid metabolism are important regulators of the acute vascular inflammatory response in vivo, and suggest that the anti-inflammatory effects of modulating each pathway may be mediated, at least in part, by overlapping mechanisms.
\end{abstract}

\section{Keywords}

CYP2J2; soluble epoxide hydrolase; cyclooxygenase; vascular inflammation; epoxyeicosatrienoic acid; prostaglandin

\section{INTRODUCTION}

\begin{abstract}
Vascular inflammation is characterized by endothelial activation and subsequent infiltration of leukocytes into the surrounding tissue [1]. Nuclear factor-kappa B (NF- $\mathrm{kB}$ ) initiates and propagates this coordinated process through transcriptional activation of cellular adhesion
\end{abstract}

\footnotetext{
(C) 2012 Elsevier Inc. All rights reserved.

Correspondence to: Craig R. Lee, Pharm.D., Ph.D., CB\# 7569, Kerr Hall, Division of Pharmacotherapy and Experimental Therapeutics, UNC Eshelman School of Pharmacy, Chapel, Hill, NC 27599-7569; Phone: 919-843-7673; Fax: 919-962-0644; craig_lee@unc.edu.

contributed equally to this work

Publisher's Disclaimer: This is a PDF file of an unedited manuscript that has been accepted for publication. As a service to our customers we are providing this early version of the manuscript. The manuscript will undergo copyediting, typesetting, and review of the resulting proof before it is published in its final citable form. Please note that during the production process errors may be discovered which could affect the content, and all legal disclaimers that apply to the journal pertain.
} 
molecules (CAMs), chemokines, and cytokines [2,3]. This fundamental pathological process plays a pivotal role in the pathogenesis of numerous acute and chronic inflammation-related diseases such as sepsis, asthma, and cardiovascular disease (CVD). Thus, identification of the key pathways that regulate NF- $\mathrm{kB}$-dependent vascular inflammatory responses offers enormous potential to facilitate the development of novel anti-inflammatory therapeutic strategies.

Eicosanoids are biologically active fatty acids derived through oxidative metabolism of arachidonic acid by the cyclooxygenase (COX), lipoxygenase (LOX), and cytochrome P450 (CYP) enzyme systems [4]. It is well established that COX-derived prostaglandins are integral regulators of inflammation, such that inhibition of COX-mediated prostaglandin biosynthesis produces potent anti-inflammatory effects in preclinical models and humans [5]. Most notably, prostaglandin E2 (PGE2) and thromboxane A2 (TxA2) are key proinflammatory products of this pathway that activate NF- $\kappa \mathrm{B}$, promote leukocyte infiltration into tissue, and thus drive the vascular inflammatory response [6,7].

In parallel, CYP epoxygenases from the CYP2J and CYP2C subfamilies catalyze the metabolism of arachidonic acid into epoxyeicosatrienoic acids (EETs), which have become increasingly recognized to possess potent anti-inflammatory properties [8]. The life of circulating EETs, however, is ephemeral as they are quickly hydrolyzed into the less potent dihydroxyeicosatrienoic acids (DHETs) by soluble epoxide hydrolase (sEH, EPHX2). We have previously shown that potentiation of CYP epoxygenase-derived EETs by endothelial CYP2J2 overexpression (Tie2-CYP2J2-Tr), endothelial CYP2C8 overexpression (Tie2$C Y P 2 C 8-\mathrm{Tr})$ or global sEH disruption $\left(E p h \times 2^{-/-}\right)$attenuates NF- $\mathrm{kB}-$ dependent acute vascular inflammatory responses in vivo [9].

Past studies have shown that inhibition of one arachidonic acid pathway may shunt metabolism down another parallel path [10,11]. Furthermore, additive attenuation of lipopolysaccharide (LPS)-induced pain and hypotension from simultaneous augmentation of CYP-derived EETs in tandem with cyclooxygenase (COX) inhibition has been recently reported $[12,13]$. However, functional interactions between the COX and CYP epoxygenase pathways in the regulation of acute vascular inflammation in vivo have not been studied. Consequently, we investigated the relative and additive effects of endothelial CYP2J2 overexpression, global sEH disruption, and pharmacologic COX inhibition on the acute vascular inflammatory response to the endotoxin LPS, a well-established activator of NFкB-dependent endothelial activation and leukocyte infiltration [14].

\section{MATERIALS AND METHODS}

\section{Chemicals}

Chemicals were purchased from Sigma-Aldrich (St. Louis, MO, USA) unless otherwise noted.

\section{Animal}

All experiments were completed in adult male and female mice on a C57BL/6 background. Transgenic (Tr) mice that express human CYP2J2 in endothelial cells under control of the murine Tie2 promoter and full enhancer (Tie2-CYP2J2-Tr) were developed on a pure C57BL/6 background, as previously described [15]. A colony of mice with targeted disruption of $E p h \times 2\left(E p h \times 2^{--}\right)$was rederived and backcrossed onto a C57BL/6 genetic background for more than 10 generations $[16,17]$. We have previously reported significantly higher endothelial EET biosynthesis in Tie2-CYP2J2-Tr mice and circulating epoxide:diol ratios in $E p h \times 2^{-/-}$mice, compared to wild-type (WT) littermates, consistent with CYP epoxygenase overexpression and sEH disruption, respectively $[9,15,17,18]$. All experiments 
with Tie2-CYP2J2-Tr mice used Tr mice from multiple founder lines and WT littermates as controls. Experiment 2, which did not involve the genetically-modified mice, utilized commercially available C57BL/6 mice (Taconic, Hudson, NY, USA). All mice had free access to food and water, and were housed in controlled conditions for temperature and humidity using a 12-hour light/dark cycle. All experiments were completed in accordance with the US National Institutes of Health (NIH) Guide for the Care and Use of Laboratory Animals and were approved by the Institutional Animal Care and Use Committee at the University of North Carolina at Chapel Hill and the National Institute of Environmental Health Sciences.

\section{Experimental Protocol}

Wild-type C57BL/6, Tie2-CYP2J2-Tr and Eph $\times 2^{-/-}$mice weighing 18-31 grams were pretreated with the nonselective COX inhibitor indomethacin $(1,2.5,5$ or $10 \mathrm{mg} / \mathrm{kg}$ ) or vehicle ( $0.5 \%$ carboxymethylcellulose) once daily for 3 days via oral gavage. On day 3 , one hour following indomethacin or vehicle administration, mice received a single dose of Escherichia coli LPS (serotype O111:B4, 1,000,000 EU/mg; $10 \mathrm{mg} / \mathrm{kg}$ ) or endotoxin-free phosphate-buffered saline (PBS) by intraperitoneal injection. Mice were euthanized by $\mathrm{CO}_{2}$ inhalation 3 hours after LPS (or PBS) administration, as previously described [9]. Blood was collected by cardiac puncture, and plasma was separated by centrifugation. Lung and liver, highly vascularized tissues that exhibit prominent activation of NF- $\kappa \mathrm{B}$ signaling and leukocyte infiltration in vivo upon LPS administration $[14,19]$ were snap-frozen in liquid nitrogen and stored at $-80^{\circ} \mathrm{C}$ for protein isolation.

\section{Myeloperoxidase Activity}

Myeloperoxidase (MPO) is a leukocyte-derived heme oxidase primarily carried by polymorphonuclear neutrophils [20]. Since MPO enzymatic activity in parenchyma predominantly reflects the presence of neutrophils, and correlates with immunohistochemical detection of neutrophil margination and tissue infiltration in this model [9], we utilized MPO functional activity as an in vivo biomarker of LPS-induced neutrophil infiltration and the primary index of vascular inflammation in these experiments. Briefly, frozen tissue $(30 \mathrm{mg}$ ) was homogenized in $20 \mathrm{mM}$ PBS with $10 \mathrm{mM} \mathrm{N}$ ethylmaleimide ( $\mathrm{pH} 7.4$ ), and centrifuged at $10,000 \mathrm{x}$ g for 15 minutes at $4^{\circ} \mathrm{C}$. The pellet was resuspended in $50 \mathrm{mM}$ PBS (pH 6.0) containing $0.5 \%$ hexadecyltrimethylammonium bromide, sonicated for 30 seconds, and centrifuged at $8,000 \mathrm{x}$ g for 20 minutes at $4^{\circ} \mathrm{C}$. MPO activity in the supernatant was quantified using the Fluorescent Myeloperoxidase Detection Kit (Cell Technology, Mountain View, CA, USA), according to the manufacturer's instructions. Fluorescence was quantified on a FLUOstar Omega Microplate Reader (IMGEN Technologies, Alexandria, VA, USA) at excitation/emission wavelengths of $530 / 590 \mathrm{~nm}$.

\section{Quantification of Eicosanoids}

Plasma epoxy (8,9-, 11,12-, 14,15-EET), dihydroxy (5,6-, 8,9-, 11,12-, 14,15-DHET), and prostaglandin (PGE2, TxB2, 6-keto-PGF1a) metabolites of arachidonic acid were quantified using an established HPLC-MS/MS method, following solid-phase extraction, as described [17,21]. The sum total of EETs plus DHETs and the 14,15-EET:DHET ratio were used as biomarkers of CYP epoxygenase and sEH metabolic function, respectively [9,17]. In a subset of experiments, plasma PGEM (a stable metabolite of PGE2) and 11-dehydro-TxB2 (a stable metabolite of TxA2) concentrations were quantified using the Prostaglandin E Metabolite and 11-dehydro Thromboxane B2 EIA kits (Cayman Chemical, Ann Arbor, MI, USA), respectively, according to the manufacturer's instructions. 


\section{Statistical Analysis}

Data were expressed as mean \pm standard error of the mean (SEM) and were normalized to the PBS-treated WT control group unless otherwise indicated. Rank-transformed mean values were compared using a one-way ANOVA followed by Fisher's LSD post hoc test. Statistical analysis was performed using SAS 9.2 (SAS Institute, Cary, NC). P $<0.05$ was considered statistically significant.

\section{RESULTS}

\section{Experiment 1: Relative effect of endothelial CYP2J2 overexpression and high-dose indomethacin}

In pilot studies, high-dose $(10 \mathrm{mg} / \mathrm{kg})$ indomethacin significantly inhibited the LPSmediated increase in plasma PGEM and 11-dehydro-TxB2 concentrations by $99 \pm 0.2 \%$ and $77 \pm 2 \%$, respectively ( $\mathrm{p}<0.05$ versus vehicle, $\mathrm{n}=6-8$ per group). The induction of lung MPO activity following LPS administration was significantly attenuated in both Tie2-CYP2J2-Tr and indomethacin $(10 \mathrm{mg} / \mathrm{kg})$-treated WT mice compared to vehicle-treated WT mice (Figure 1, $\mathrm{p}<0.05$ for each comparison). Furthermore, high-dose indomethacin attenuated the induction of MPO activity to a significantly greater degree than observed in Tie2CYP2J2-Tr mice $(67 \pm 9 \%$ versus $37 \pm 11 \%$, respectively, $\mathrm{p}<0.05)$.

\section{Experiment 2: Relative effect of incremental indomethacin doses}

A dose-response experiment was conducted in order to identify an indomethacin dose that attenuated LPS-induced vascular inflammation to a similar degree as genetic potentiation of the CYP epoxygenase pathway. LPS-mediated induction of plasma PGEM (Figure 2A) and lung MPO activity (Figure 2B) was attenuated by indomethacin in a dose-dependent manner. Induction of liver MPO activity was also significantly attenuated by indomethacin at each dose compared to vehicle (Figure 2C); however, a dose-dependent attenuation was not observed. Since indomethacin $2.5 \mathrm{mg} / \mathrm{kg}$ produced a $38 \pm 5 \%$ attenuation of LPS-induced lung MPO activity, similar to the $37 \pm 11 \%$ attenuation observed in Tie2-CYP2J2-Tr mice from Experiment 1 and the attenuation observed in both Tie2-CYP2J2-Tr and Ephx $2^{-/-}$ mice from prior studies [9], this "moderate" dose of indomethacin was utilized in subsequent experiments to evaluate the potential additive effect of simultaneously modulating both pathways.

\section{Experiment 3: Relative and additive effect of endothelial CYP2J2 overexpression, global sEH disruption, and moderate-dose indomethacin}

LPS-induction of lung MPO activity was significantly attenuated in indomethacin $(2.5 \mathrm{mg} /$

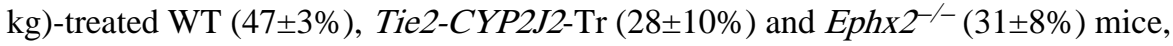
compared to vehicle-treated WT controls (Figure 3A, $\mathrm{p}<0.05$ for each comparison). MPO

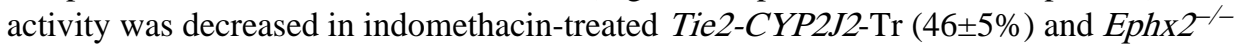
$(48 \pm 4 \%)$ mice to a level similar to that observed in indomethacin-treated WT mice; however, a significant additive anti-inflammatory effect was not observed (Figure 3A, $\mathrm{p}=0.848$ and $\mathrm{p}=0.851$, respectively, versus indomethacin-treated WT mice). Similar results across genotype and treatment groups were observed following LPS-induction of liver MPO activity (Figure 3B), although the observed attenuation in vehicle and indomethacin-treated Tie2-CYP2J2-Tr mice was not statistically significant ( $\mathrm{p}=0.397$ and $\mathrm{p}=0.054$, respectively, versus vehicle-treated WT mice).

Indomethacin markedly inhibited the LPS-mediated induction of plasma PGEM, TxB2, and 6-keto-PGF1a levels in WT, Tie2-CYP2J2-Tr, and Ephx $2^{-/-}$mice (Figure 4A-C, p<0.05 versus vehicle in each genotype group). In contrast, no differences were observed across genotype within the vehicle or indomethacin-treated groups (Figure 4A-C). Similar results 
were observed with plasma PGD2 and PGF2a levels, and no treatment or genotype differences in hepatic or pulmonary Ptgs 2 mRNA levels were observed (data not shown). The total sum of EET+DHET levels was approximately 1.3-fold higher in Tie2-CYP2J2-Tr mice compared to WT ( $\mathrm{p}=0.064)$ and $E p h \times 2^{-/-}$(p=0.047) mice (Figure 4D), consistent with prior studies [9,18]. The 14:15-EET:DHET ratio was drastically increased in Ephx $2^{-/-}$mice compared to WT and Tie2-CYP2J2-Tr mice, irrespective of treatment (Figure 4E, p $<0.05$ for all comparisons). Indomethacin did not significantly alter the total sum of EET+DHET levels in Tie2-CYP2J2-Tr mice ( $\mathrm{p}=0.092$ versus vehicle) or the 14:15-EET:DHET ratio in $E p h \times 2^{-/-}$mice ( $=0.596$ versus vehicle). Furthermore, no treatment or genotype differences in 5-LOX-derived 5-hydroxyeicosatetraenoic acid (5-HETE) or CYP $\omega$-hydroxylase-derived 20-HETE levels were observed (data not shown).

\section{DISCUSSION}

Functional characterization of the key eicosanoid metabolism pathways that regulate vascular inflammatory responses in vivo offers enormous potential to facilitate the development of new anti-inflammatory therapeutic strategies. Consequently, we investigated the relative and additive effects of endothelial CYP2J2 overexpression, global sEH disruption, and pharmacologic COX inhibition on LPS-induced acute vascular inflammation in mice. Our studies demonstrate that: 1) high-dose indomethacin attenuates acute vascular inflammation to a significantly greater extent than genetic potentiation of the CYP epoxygenase pathway; 2) moderate-dose indomethacin attenuates acute vascular inflammation to a similar degree as either endothelial CYP2J2 overexpression or global sEH disruption; and, 3) dual modulation of the COX and CYP epoxygenase pathways does not additively attenuate the acute vascular inflammatory response to LPS. Collectively, these findings demonstrate that both COX and CYP epoxygenase-mediated eicosanoid metabolism are important regulators of the acute vascular inflammatory response in vivo, and suggest that the anti-inflammatory effects of modulating each pathway may be mediated, at least in part, by overlapping mechanisms.

Consistent with our prior studies and hypothesis, the acute vascular inflammatory response to LPS was significantly and similarly attenuated in Tie2-CYP2J2-Tr and Eph $2^{-/-}$mice [9], further demonstrating that increased endothelial EET biosynthesis and decreased sEHmediated EET hydrolysis elicit potent anti-inflammatory effects in the vasculature in vivo. Similarly, inhibition of COX-mediated prostaglandin biosynthesis with the nonselective COX inhibitor indomethacin significantly attenuated vascular inflammation, consistent with prior studies in various models of inflammation [5]. More specifically, administration of a moderate $(2.5 \mathrm{mg} / \mathrm{kg} /$ day $)$ dose of indomethacin elicited an approximate $80 \%$ reduction in LPS-induced plasma PGEM levels and a reduction in pulmonary and hepatic MPO activity equivalent to that observed in Tie2-CYP2J2-Tr and Ephx $2^{-/-}$mice. Administration of high $(10 \mathrm{mg} / \mathrm{kg} /$ day $)$ dose indomethacin, however, produced a complete abrogation of LPSinduced PGE2 biosynthesis and a significantly greater anti-inflammatory effect than genetic potentiation of the CYP epoxygenase pathway. These results suggest that maximizing inhibition of COX-mediated prostaglandin biosynthesis yields a greater anti-inflammatory effect than maximizing inhibition of EET hydrolysis via genetic disruption of Ephx2. Importantly, administration of high doses of nonselective COX inhibitors such as indomethacin produces substantial, and often intolerable, dose-dependent adverse effects. Consequently, identifying therapeutic strategies that enhance the anti-inflammatory effects of lower doses could expand the therapeutic window of COX inhibitors.

Arachidonic acid serves as a common substrate for the parallel biosynthesis of biologically active COX-, LOX- and CYP-derived eicosanoids. Two recent reports have demonstrated that $\mathrm{COX}$ and sEH inhibition can shunt arachidonic acid metabolism down parallel 
metabolic paths $[10,11]$. Thus, shunting metabolism away from pro-inflammatory COXderived prostaglandins and toward anti-inflammatory CYP-derived EETs would be hypothesized to yield a greater anti-inflammatory effect than modulation of either pathway alone. Indeed, an additive attenuation of LPS-induced pain [13] and hypotension [12] following dual inhibition of COX and SEH has been reported, suggesting that functional interactions exist between COX- and CYP epoxygenase-mediated arachidonic acid metabolism (i.e., where modulation of one pathway enhances the effects of the other). The effects of COX inhibition in tandem with potentiation of CYP-derived EETs on vascular inflammation, however, had not been evaluated to date. The current investigation demonstrates that despite anti-inflammatory effects following modulation of each pathway alone, simultaneous inhibition of COX-derived prostaglandin biosynthesis and potentiation of CYP epoxygenase-derived EET levels does not additively attenuate the acute vascular inflammatory response to LPS in vivo. Although indomethacin has been previously reported to inhibit CYP epoxygenase activity in vitro [22], the observed IC50 in these experiments $(70 \mu \mathrm{M})$ was more than 20 -fold above the peak plasma concentrations following high dose $(10 \mathrm{mg} / \mathrm{kg})$ treatment in rodents [23]. In the current investigation, in vivo EET levels were not significantly suppressed by indomethacin in WT, Tie2-CYP2J2-Tr or Ephx $2^{-/-}$mice. Moreover, an increase in metabolism down the parallel 5-LOX and CYP $\omega$-hydroxylase pathways was not observed. Thus, the observed lack of an additive anti-inflammatory effect was not secondary to indomethacin-mediated inhibition of EET biosynthesis or shunting arachidonic acid metabolism down a parallel pro-inflammatory pathway. Although the specific mechanism remains unclear, these data collectively suggest that the vascular antiinflammatory effects of COX inhibition and increasing EET levels may be mediated by an overlapping mechanism.

Prior evidence has demonstrated the presence of metabolic and functional crosstalk between the CYP epoxygenase and COX pathways, independent of arachidonic acid substrate shunting. Most notably, EETs have been reported to inhibit COX enzymatic activity in ram seminal vesicles, rat monocytes and murine vascular smooth muscle cells in vitro [24-26], suppress LPS-induction of hepatic and spinal COX-2 expression in vivo [13,27,28], and consequently attenuate the induction of pro-inflammatory prostaglandin biosynthesis [13,25-28]. These observations suggest that inhibition of prostaglandin biosynthesis may contribute to the anti-inflammatory effects of EETs. In the presence of genetic potentiation of EET levels, however, we did not observe any significant differences in prostaglandin levels. Similar results were recently reported following induction of tumor growth in these genetically-modified mice [18]. Collectively, this suggests that EET-mediated attenuation of prostaglandin biosynthesis does not contribute to the acute vascular anti-inflammatory phenotype observed in Tie2-CYP2J2-Tr and Ephx $2^{-/-}$mice.

It has also been reported that EETs induce COX-2 expression and prostacyclin production in cultured endothelial cells in the absence of an inflammatory stimulus [29], suggesting that EET-mediated modulation of prostaglandin biosynthesis may be dependent on the specific model or cell type under investigation. Endotoxin-induced hypotension and nociception are phenotypes primarily driven by inducible nitric oxide synthase-mediated vasodilation [30] and cAMP-mediated signaling [27,31], respectively, as compared to induction of CAM and chemokine-mediated endothelial activation and leukocyte infiltration into tissue [14]. Furthermore, the hypotension and pain phenotypes that were additively attenuated by dual modulation of the COX and CYP epoxygenase pathways were assessed 6 hours following LPS administration [12,13]. In contrast, we measured MPO activity, an established biomarker of neutrophil infiltration, 3 hours following LPS administration [9]. Since induction of inflammatory CAM, chemokine and cytokine expression peaks 2-4 hours following administration of LPS [32,33], functional interactions between EET and prostaglandin biosynthesis may be unmasked at later time points during resolution of the 
acute inflammatory response. These findings collectively highlight the complexity of initiation and resolution of the acute inflammatory response, and the potential contribution of temporal and phenotypic differences to the role of eicosanoid metabolism in the regulation of inflammation.

More recently, EETs have been reported to selectively antagonize recombinant Tx (TP) receptors, with little or no binding affinity for non-TP prostanoid or leukotriene receptors, and specifically inhibit TP receptor agonist-mediated constriction of isolated rodent resistance arteries, conduit arteries and bronchi [34]. In accordance, EETs also blocked TP receptor agonist-mediated constriction of isolated human bronchi, while administration of a CYP epoxygenase inhibitor increased TP-dependent bronchoconstriction [35]. Additionally, EETs inhibit arachidonic acid-stimulated platelet aggregation in isolated human platelets without changing Tx levels [24], further corroborating the potential presence of redundancy in the biological effects of increasing CYP-derived EET and decreasing COX-derived TxA2 levels. Importantly, activation of TP-receptor signaling also drives acute and chronic vascular inflammatory responses, including cytokine-mediated activation of leukocyte adhesion [7] and diabetes-induced atherosclerosis [36]. Considering the lack of an additive anti-inflammatory effect following dual modulation of the COX and CYP epoxygenase pathways in the current investigation, it is conceivable that attenuation of TP receptor signaling contributes, at least in part, to the vascular anti-inflammatory effects of both COX inhibition (via inhibiting TxA2 biosynthesis) and increasing CYP-derived EETs (via competitive inhibition of TP receptors). These observations collectively highlight the importance for future studies to elucidate functional interactions between EETs and TP receptor signaling in the regulation of vascular inflammation. Furthermore, thoroughly understanding the relative roles of, functional interactions between and mechanisms underlying the parallel CYP epoxygenase, CYP $\omega$-hydroxylase, COX (including the specific prostaglandin synthases and receptors), and LOX pathways of arachidonic acid metabolism in the regulation of acute and chronic vascular inflammatory responses will be essential in order to maximize the anti-inflammatory therapeutic potential of targeting eicosanoid metabolism.

\section{CONCLUSIONS}

Although potentiation of the CYP epoxygenase pathway, through increased EET biosynthesis or decreased EET hydrolysis, and non-selective inhibition of COX-mediated prostaglandin biosynthesis each attenuated the acute vascular inflammatory response to endotoxin in vivo, dual modulation of both pathways did not result in an additive antiinflammatory effect. These findings further demonstrate that both the COX and CYP epoxygenase pathways are important regulators of vascular inflammation, and suggest that the anti-inflammatory effect of modulating each pathway may be mediated, at least in part, by overlapping mechanisms. Future studies delineating the complex functional interactions between these parallel metabolic pathways in the regulation of inflammation are necessary.

\section{Acknowledgments}

This publication was made possible by a University of North Carolina at Chapel Hill Chancellor's Fellowship to Dr. Oni-Orisan, a predoctoral training program in Integrative Vascular Biology (T32 HL069768) and predoctoral fellowship from the American Heart Association to Dr. Schuck, a predoctoral fellowship from the American Foundation for Pharmaceutical Education to Dr. Theken, funds from the Intramural Research Program of the NIH/ NIEHS to Dr. Tomer (Z01 ES050167) and Dr. Zeldin (Z01 ES025034), and grants R01 GM088199 and R01 GM088199-S1 to Dr. Lee. Its contents are solely the responsibility of the authors and do not necessarily represent the official views of the NIH. 


\section{References}

1. Lawrence T, Willoughby DA, Gilroy DW. Anti-inflammatory lipid mediators and insights into the resolution of inflammation. Nat Rev Immunol. 2002; 2:787-95. [PubMed: 12360216]

2. Liu SF, Malik AB. NF- $\kappa$ B activation as a pathological mechanism of septic shock and inflammation. Am J Physiol Lung Cell Mol Physiol. 2006; 290:L622-45. [PubMed: 16531564]

3. Barnes PJ, Karin M. Nuclear factor-kappaB: A pivotal transcription factor in chronic inflammatory diseases. N Engl J Med. 1997; 336:1066-71. [PubMed: 9091804]

4. Buczynski MW, Dumlao DS, Dennis EA. Thematic review series: Proteomics. an integrated omics analysis of eicosanoid biology. J Lipid Res. 2009; 50:1015-38. [PubMed: 19244215]

5. Ricciotti E, FitzGerald GA. Prostaglandins and inflammation. Arterioscler Thromb Vasc Biol. 2011; 31:986-1000. [PubMed: 21508345]

6. Poligone B, Baldwin AS. Positive and negative regulation of NF- $\kappa$ B by COX-2. J Biol Chem. 2001; 276:38658-64. [PubMed: 11509575]

7. Katagiri H, Ito Y, Ito S, Murata T, Yukihiko S, Narumiya S, et al. TNF-alpha induces thromboxane receptor signaling-dependent microcirculatory dysfunction in mouse liver. Shock. 2008; 30:463-7. [PubMed: 18800000]

8. Deng Y, Theken KN, Lee CR. Cytochrome P450 epoxygenases, soluble epoxide hydrolase, and the regulation of cardiovascular inflammation. J Mol Cell Cardiol. 2010; 48:331-41. [PubMed: 19891972]

9. Deng Y, Edin ML, Theken KN, Schuck RN, Flake GP, Kannon MA, et al. Endothelial CYP epoxygenase overexpression and soluble epoxide hydrolase disruption attenuate acute vascular inflammatory responses in mice. FASEB J. 2011; 25:703-13. [PubMed: 21059750]

10. Maxis K, Delalandre A, Martel-Pelletier J, Pelletier JP, Duval N, Lajeunesse D. The shunt from the cyclooxygenase to lipoxygenase pathway in human osteoarthritic subchondral osteoblasts is linked with a variable expression of the 5-lipoxygenase-activating protein. Arthritis Res Ther. 2006; 8:R181. [PubMed: 17156456]

11. Jung O, Jansen F, Mieth A, Barbosa-Sicard E, Pliquett RU, Babelova A, et al. Inhibition of the soluble epoxide hydrolase promotes albuminuria in mice with progressive renal disease. PLoS ONE. 2010; 5:e11979. [PubMed: 20694143]

12. Liu J, Yang J, Inceoglu B, Qiu H, Ulu A, Hwang S, et al. Inhibition of soluble epoxide hydrolase enhances the anti-inflammatory effects of aspirin and 5-lipoxygenase activation protein inhibitor in a murine model. Biochem Pharmacol. 2010; 79:880-7. [PubMed: 19896470]

13. Schmelzer KR, Inceoglu B, Kubala L, Kim I, Jinks SL, Eiserich JP, et al. Enhancement of antinociception by coadministration of nonsteroidal anti-inflammatory drugs and soluble epoxide hydrolase inhibitors. Proc Natl Acad Sci U S A. 2006; 103:13646-51. [PubMed: 16950874]

14. Kerfoot SM, Kubes P. Local coordination verses systemic disregulation: Complexities in leukocyte recruitment revealed by local and systemic activation of TLR4 in vivo. J Leukoc Biol. 2005; 77:862-7. [PubMed: 15743884]

15. Lee CR, Imig JD, Edin ML, Foley J, DeGraff LM, Bradbury JA, et al. Endothelial expression of human cytochrome P450 epoxygenases lowers blood pressure and attenuates hypertensioninduced renal injury in mice. FASEB J. 2010; 24:3770-81. [PubMed: 20495177]

16. Sinal CJ, Miyata M, Tohkin M, Nagata K, Bend JR, Gonzalez FJ. Targeted disruption of soluble epoxide hydrolase reveals a role in blood pressure regulation. J Biol Chem. 2000; 275:40504-10. [PubMed: 11001943]

17. Seubert JM, Sinal CJ, Graves J, DeGraff LM, Bradbury JA, Lee CR, et al. Role of soluble epoxide hydrolase in postischemic recovery of heart contractile function. Circ Res. 2006; 99:442-50. [PubMed: 16857962]

18. Panigrahy D, Edin ML, Lee CR, Huang S, Bielenberg DR, Butterfield CE, et al. Epoxyeicosanoids stimulate multiorgan metastasis and tumor dormancy escape in mice. J Clin Invest. 2012; 122:178-91. [PubMed: 22182838]

19. Carlsen H, Moskaug J, Fromm SH, Blomhoff R. In vivo imaging of NF- $\kappa B$ activity. J Immunol. 2002; 168:1441-6. [PubMed: 11801687] 
20. Lau D, Baldus S. Myeloperoxidase and its contributory role in inflammatory vascular disease. Pharmacol Ther. 2006; 111:16-26. [PubMed: 16476484]

21. Edin ML, Wang Z, Bradbury JA, Graves JP, Lih FB, DeGraff LM, et al. Endothelial expression of human cytochrome $\mathrm{P} 450$ epoxygenase CYP2C8 increases susceptibility to ischemia-reperfusion injury in isolated mouse heart. FASEB J. 2011; 25:3436-47. [PubMed: 21697548]

22. Capdevila J, Gil L, Orellana M, Marnett LJ, Mason JI, Yadagiri P, et al. Inhibitors of cytochrome P-450-dependent arachidonic acid metabolism. Arch Biochem Biophys. 1988; 261:257-63. [PubMed: 3128168]

23. Kim SY, Lee YM, Shin HJ, Kang JS. Indomethacin-loaded methoxy poly(ethylene glycol)/poly( $\varepsilon$ caprolactone) diblock copolymeric nanosphere: Pharmacokinetic characteristics of indomethacin in the normal Sprague-Dawley rats. Biomaterials. 2001; 22:2049-56. [PubMed: 11426885]

24. Fitzpatrick FA, Ennis MD, Baze ME, Wynalda MA, McGee JE, Liggett WF. Inhibition of cyclooxygenase activity and platelet aggregation by epoxyeicosatrienoic acids. influence of stereochemistry. J Biol Chem. 1986; 261:15334-8. [PubMed: 3095326]

25. Kozak W, Aronoff DM, Boutaud O, Kozak A. 11,12-epoxyeicosatrienoic acid attenuates synthesis of prostaglandin E2 in rat monocytes stimulated with lipopolysaccharide. Exp Biol Med. 2003; 228:786-94.

26. Fang X, Moore SA, Stoll LL, Rich G, Kaduce TL, Weintraub NL, et al. 14,15-epoxyeicosatrienoic acid inhibits prostaglandin E2 production in vascular smooth muscle cells. Am J Physiol. 1998; 275:H2113-21. [PubMed: 9843811]

27. Inceoglu B, Jinks SL, Ulu A, Hegedus CM, Georgi K, Schmelzer KR, et al. Soluble epoxide hydrolase and epoxyeicosatrienoic acids modulate two distinct analgesic pathways. Proc Natl Acad Sci U S A. 2008; 105:18901-6. [PubMed: 19028872]

28. Schmelzer KR, Kubala L, Newman JW, Kim I, Eiserich JP, Hammock BD. Soluble epoxide hydrolase is a therapeutic target for acute inflammation. Proc Natl Acad Sci U S A. 2005; 102:9772-7. [PubMed: 15994227]

29. Michaelis UR, Falck JR, Schmidt R, Busse R, Fleming I. Cytochrome P4502C9-derived epoxyeicosatrienoic acids induce the expression of cyclooxygenase-2 in endothelial cells. Arterioscler Thromb Vasc Biol. 2005; 25:321-6. [PubMed: 15569819]

30. Calvert TJ, Chicoine LG, Liu Y, Nelin LD. Deficiency of mitogen-activated protein kinase phosphatase-1 results in iNOS-mediated hypotension in response to low-dose endotoxin. Am J Physiol Heart Circ Physiol. 2008; 294:H1621-9. [PubMed: 18281381]

31. Inceoglu B, Wagner K, Schebb NH, Morisseau C, Jinks SL, Ulu A, et al. Analgesia mediated by soluble epoxide hydrolase inhibitors is dependent on cAMP. Proc Natl Acad Sci U S A. 2011; 108:5093-7. [PubMed: 21383170]

32. Copeland S, Warren HS, Lowry SF, Calvano SE, Remick D. the Inflammation and the Host Response to Injury Investigators. Acute inflammatory response to endotoxin in mice and humans. Clin Diagn Lab Immunol. 2005; 12:60-7. [PubMed: 15642986]

33. Rojas M, Woods CR, Mora AL, Xu J, Brigham KL. Endotoxin-induced lung injury in mice: Structural, functional, and biochemical responses. Am J Physiol Lung Cell Mol Physiol. 2005; 288:L333-41. [PubMed: 15475380]

34. Behm DJ, Ogbonna A, Wu C, Burns-Kurtis CL, Douglas SA. Epoxyeicosatrienoic acids function as selective, endogenous antagonists of native thromboxane receptors: Identification of a novel mechanism of vasodilation. J Pharmacol Exp Ther. 2009; 328:231-9. [PubMed: 18836067]

35. Senouvo FY, Tabet Y, Morin C, Albadine R, Sirois C, Rousseau E. Improved bioavailability of epoxyeicosatrienoic acids reduces TP-receptor agonist-induced tension in human bronchi. Am J Physiol Lung Cell Mol Physiol. 2011; 301:L675-82. [PubMed: 21821730]

36. Zuccollo A, Shi C, Mastroianni R, Maitland-Toolan KA, Weisbrod RM, Zang M, et al. The thromboxane A2 receptor antagonist S18886 prevents enhanced atherogenesis caused by diabetes mellitus. Circulation. 2005; 112:3001-8. [PubMed: 16260636] 


\section{Highlights}

- CYP2J2 overexpression and sEH disruption each attenuate acute vascular inflammation.

- Moderate dose indomethacin attenuates vascular inflammation to a similar degree.

- Modulation of both pathways did not produce an additive anti-inflammatory effect.

- These anti-inflammatory effects may be mediated by overlapping mechanisms. 

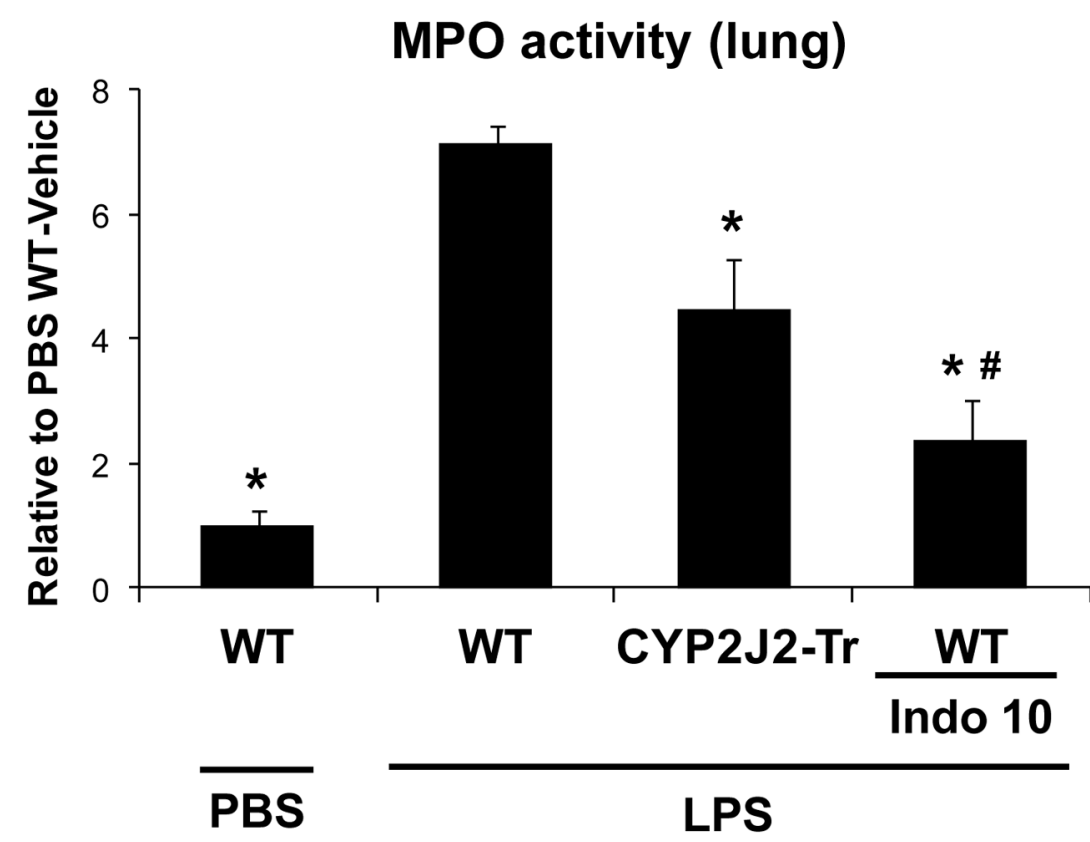

Figure 1. Relative effect of endothelial CYP2J2 overexpression and high-dose indomethacin on acute vascular inflammation

LPS-induced lung MPO activity in indomethacin- $(10 \mathrm{mg} / \mathrm{kg})$ and vehicle-treated wild-type (WT) mice and Tie2-CYP2J2-Tr mice. Data are expressed relative to saline (PBS)-treated WT controls. $\mathrm{N}=6-8$ per group. ${ }^{*} \mathrm{P}<0.05$ versus LPS WT-vehicle. ${ }^{*} \mathrm{P}<0.05$, indomethacin versus CYP2J2-Tr. 
A

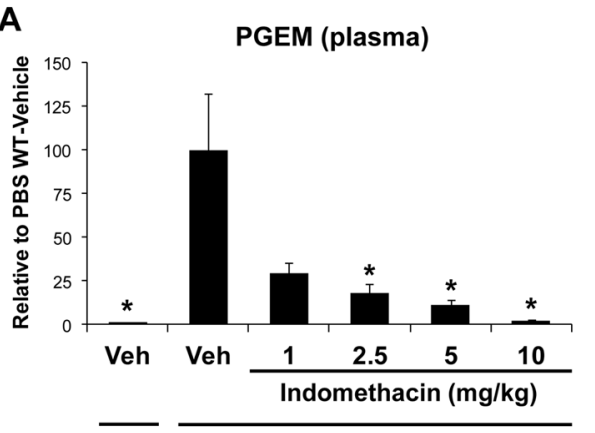

$\overline{\text { PBS }}$

$c$

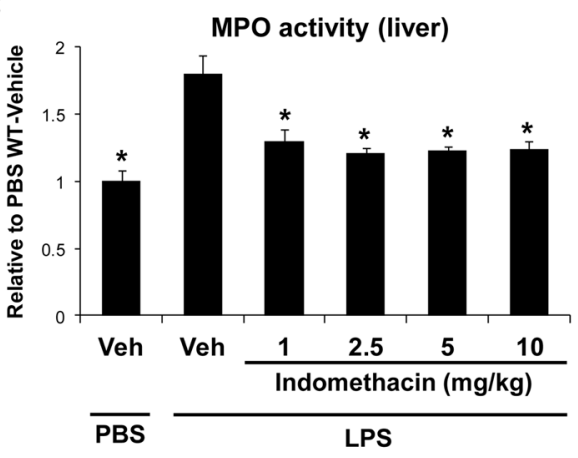

B

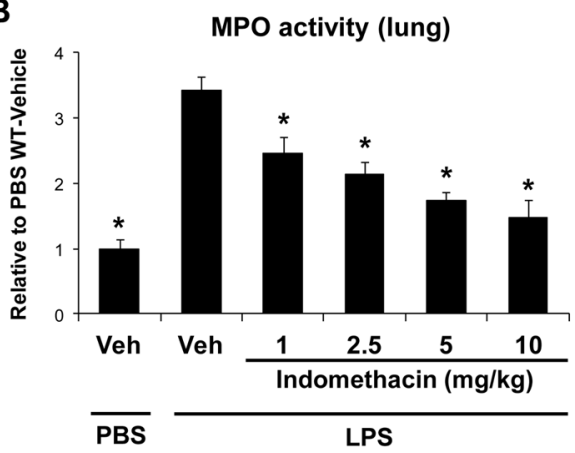

Figure 2. Dose-dependent inhibition of acute vascular inflammation by indomethacin LPS-induced (A) plasma PGEM levels, (B) lung MPO activity, (C) and liver MPO activity following administration of vehicle (Veh) or incremental doses of indomethacin $(1,2.5,5$ or $10 \mathrm{mg} / \mathrm{kg}$ ) to wild-type C57BL/6 mice. Data are expressed relative to saline (PBS) and Vehtreated controls. The mean PGEM levels in the PBS control group and LPS-treated vehicle group were 6.6 and $658 \mathrm{pg} / \mathrm{mL}$, respectively. $\mathrm{N}=4-9$ per group. ${ }^{*} \mathrm{P}<0.05$ versus LPSvehicle. 
A

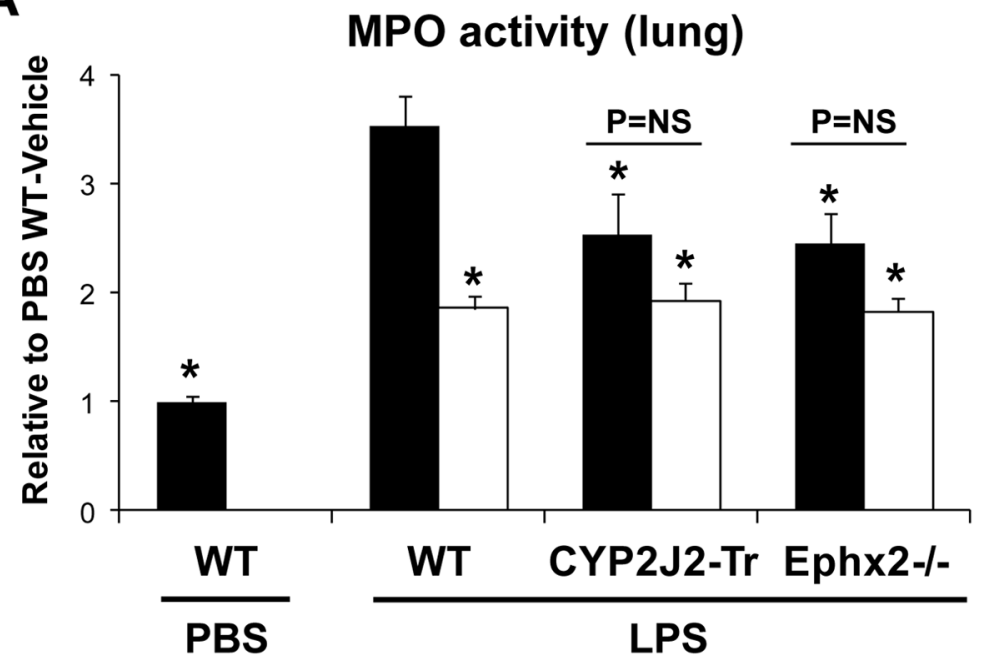

B

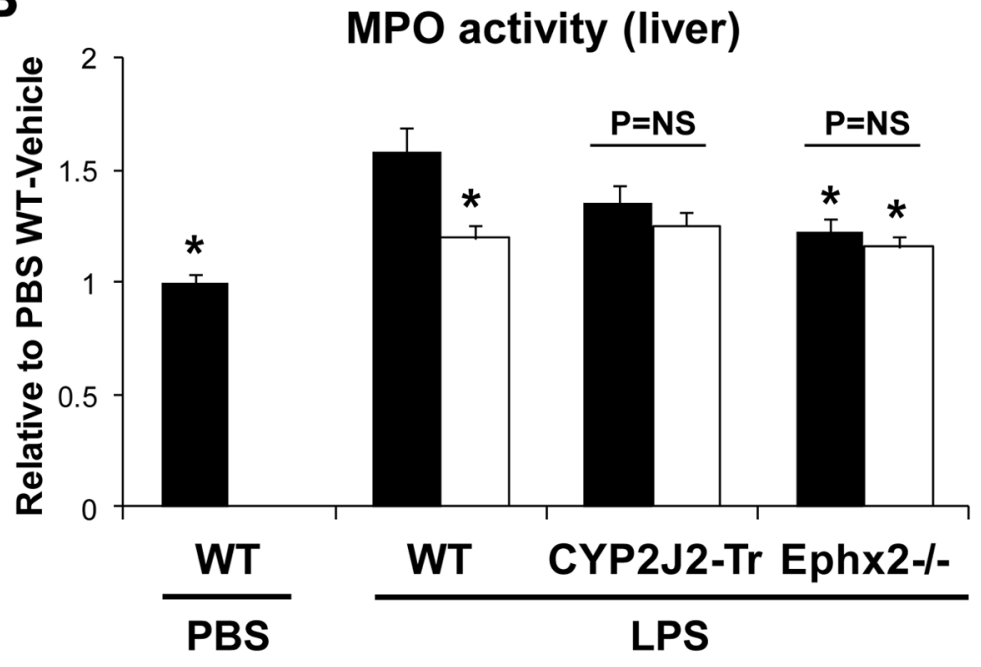

$\square$ Vehicle $\square$ Indomethacin $(2.5 \mathrm{mg} / \mathrm{kg})$

Figure 3. Relative effect of endothelial CYP2J2 overexpression, global sEH disruption, and moderate-dose indomethacin on acute vascular inflammation

LPS-induced (A) lung MPO activity and (B) liver MPO activity following administration of vehicle or indomethacin $(2.5 \mathrm{mg} / \mathrm{kg})$ to wild-type (WT), Tie2-CYP2J2-Tr, and Ephx $2^{-/-}$ mice. Data are expressed relative to saline (PBS)-treated WT controls. PBS: $N=6$ per group, LPS: $\mathrm{N}=9-18$ per group. $* \mathrm{P}<0.05$ versus LPS WT-vehicle. NS=not significant. 
A

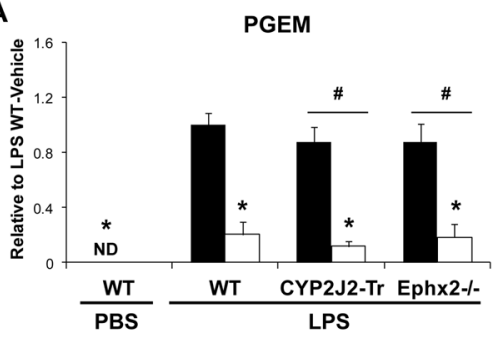

C

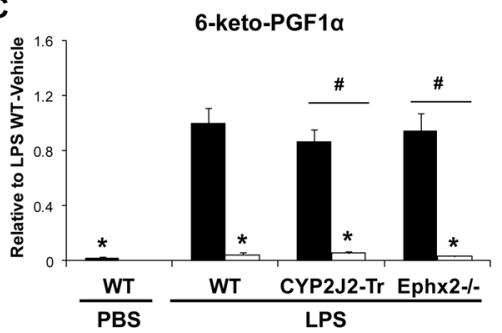

E

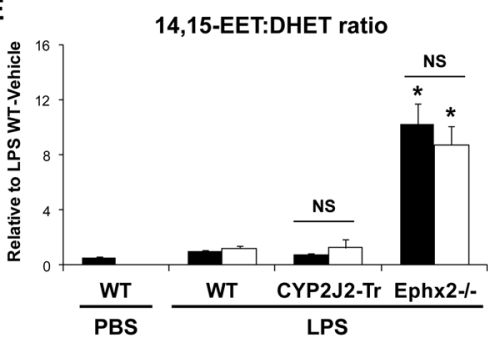

B

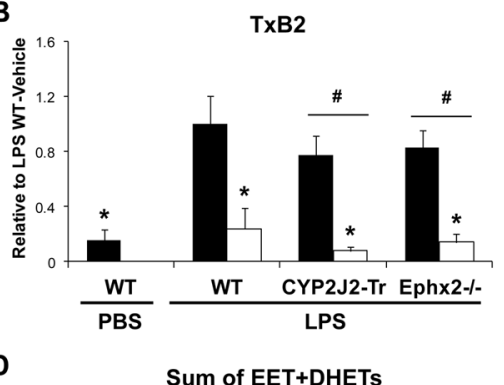

D

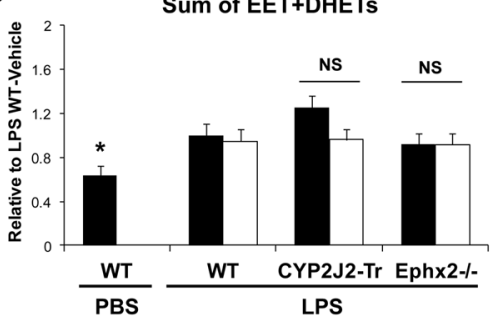

Figure 4. Relative effect of endothelial CYP2J2 overexpression, global SEH disruption, and moderate-dose indomethacin on eicosanoid metabolite levels

LPS-induced plasma (A) PGEM, (B) TxB2, and (C) 6-keto-PGF1a levels, (D) the total sum of EET+DHETs, and (E) the 14,15-EET:DHET ratio following administration of vehicle or indomethacin $(2.5 \mathrm{mg} / \mathrm{kg})$ to wild-type (WT), Tie2-CYP2J2-Tr, and Ephx $2^{-/-}$mice. The mean eicosanoid levels for the PBS control group are as follows: (A) PGEM, below the limit of detection (the mean level in the LPS-treated WT group is $379 \mathrm{pg} / \mathrm{mL}$ ); (B) TxB2, $214 \mathrm{pg}$ / mL; (C) 6-keto-PGF1a, $49.5 \mathrm{pg} / \mathrm{mL}$; (D) sum of EET+DHETs, $13.7 \mathrm{ng} / \mathrm{mL}$; (E) 14,15EET:DHET, 0.035 (ratio). PBS: $\mathrm{N}=5$ per group, LPS: $\mathrm{N}=9-16$ per group. $* \mathrm{P}<0.05$ versus LPS WT-vehicle. ${ }^{*} \mathrm{P}<0.05$, indomethacin versus vehicle within the Tie2-CYP2J2-Tr and $E p h \times 2^{-/-}$genotype groups. ND=below the limit of detection. NS=not significant. 